\title{
A Fuzzy-based Call Admission Control Scheme for Wireless Cellular Networks Considering Priority of On-going Connections
}

\author{
Gjergji Mino $^{\dagger}$, Leonard Barolli ${ }^{\ddagger}$, Arjan Durresi ${ }^{\dagger \dagger}$, Fatos Xhafa ${ }^{\dagger \ddagger}$, Akio Koyama ${ }^{\ddagger \ddagger}$ \\ $\dagger$ Graduate School of Engineering \\ Fukuoka Institute of Technology (FIT) \\ 3-30-1 Wajiro-Higashi, Higashi-Ku, Fukuoka 811-0295, Japan \\ E-mail: gjmino@gmail.com \\ ${ }^{\ddagger}$ Department of Information and Communication Engineering \\ Fukuoka Institute of Technology (FIT) \\ 3-30-1 Wajiro-Higashi, Higashi-ku, Fukuoka 811-0295, Japan \\ E-mail: barolli@fit.ac.jp \\ $\dagger \dagger$ Department of Computer and Information Science \\ Indiana University Purdue University at Indianapolis (IUPUI) \\ 723 W. Michigan Street SL 280, Indianapolis, IN 46202, USA \\ E-mail: durresi@cs.iupui.edu \\ ${ }^{\dagger}$ Department of Languages and Informatics Systems \\ Technical University of Catalonia \\ Jordi Girona 1-3, 08034 Barcelona, Spain \\ E-mail: fatos@1si.upc.edu \\ ${ }_{\ddagger}$ Department of Informatics, Yamagata University \\ 4-3-16 Jonan, Yonezawa 992-8510, Yamagata, Japan \\ E-mail: akoyama@yz.yamagata-u.ac.jp
}

\begin{abstract}
The mobile cellular systems are expected to support multiple services with guaranteed Quality of Service (QoS). But, the ability of wireless systems to accommodate expected growth of traffic load and broadband services is limited by available radio frequency spectrum. Call Admission Control (CAC) is one of the resource management functions, which regulates network access to ensure QoS provisioning. However, the decision for CAC is very challenging issue due to user mobility, limited radio spectrum, and multimedia traffic characteristics. In our previous work, we proposed a fuzzy-based CAC system and compared the performance of the proposed system with Shadow Cluster Concept (SCC). In this work, we extend our work by considering the priority of the on-going connections. We evaluate by simulations the performance of the proposed system and compare its performance with our previous work. The performance evaluation shows that the proposed system has a good behavior in keeping the QoS of on-going connections.
\end{abstract}

\section{Introduction}

The future telecommunications networks (such as the third-generation and forth-generation wireless networks) aim to provide integrated services such as voice, data, and multimedia via inexpensive low-powered mobile computing devices over wireless infrastructures. As the demand for multimedia services over the air has been steadily increasing over the last few years, wireless multimedia networks have been a very active research area [1]. To support various integrated services with a certain Quality of Service (QoS) requirement in these wireless networks, resource provisioning is a major issue.

To guarantee the QoS, Call Admission Control (CAC) is a good strategy. CAC is a provisioning strategy that limits the number of connections into the network in order to reduce the network congestion and call dropping.

$\mathrm{CAC}$ is not a problem that is unique to wireless networks. It is applicable to almost every type of networks, but in cellular wireless networks due to users' mobility the CAC becomes much more complicated. While in wired networks 
the resources are reserved for the call at set-up time and are not changed after that, in cellular wireless networks when the mobile node moves from one cell to another one, the bandwidth must be requested in the new cell. During this process, the call may not be able to get a channel in the new cell to continue its service due to the limited resource in wireless networks, which will lead to the call dropping. Thus, the new and handoff calls have to be treated differently in terms of resource allocation. Since users are much more sensitive to call dropping than to call blocking, the handoff calls are assigned higher priority than new calls [2].

$\mathrm{CAC}$ techniques are required to guarantee that all traffic types meet their QoS requirements. In order to improve the system performance at the call level (fairness in blocking), a CAC strategy may block additional calls even if there are enough resources for their service. CAC is based on the knowledge of the statistical characteristics of ongoing and arriving calls [2]. The decision to accept an additional call involves the calculation or estimation of the consequences of the call acceptance on blocking and delay of itself and other incoming calls [3].

Several schemes have been proposed for CAC in wireless cellular networks. However, during the complexity of CAC in wireless environment, many simplified models and assumptions are made. Some schemes consider that each mobile node will make hand-over to neighboring cells with equal probability, which may be not accurate in general. For this reason, the intelligent and heuristic methods are needed.

Use of intelligent methods based on Fuzzy Logic (FL), Neural Networks (NN) and Genetic Algorithms (GA) can prove to be efficient for traffic control in telecommunication networks [4-13].

In our previous work, in order to deal with CAC in wireless cellular networks, we proposed a CAC scheme based on FL $[14,15]$. We implemented and evaluated the proposed system by comparing its performance with Shadow Cluster Concept (SCC) [16].

In this paper, we extend our work by considering the priority of the on-going connections. We evaluate by simulations the proposed system and compare its performance with our previous work.

The structure of this paper is as follows. In Section 2, we present the previous work. In Section 3, we introduce the proposed system. In Section 4, we discuss the simulation results. Finally, some conclusions are given in Section 5.

\section{Previous Work}

One of the previous work on CAC is SCC [16]. The fundamental idea of the SCC is that every mobile terminal with an active wireless connection exerts an influence upon the cells (and their BSs) in the vicinity of its current location and along its direction of travel. As an active mobile terminal travels to other cells, the region of influence also moves, following the active mobile terminal to its new location. The BSs (and their cells) currently being influenced are said to form a shadow cluster, because the region of influence follows the movements of the active mobile terminal like a shadow, as shown in Fig. 1. The shadow (and therefore the level of influence) is strongest near the active mobile terminal, and fades away depending on factors such as the distance to the mobile terminal, current call holding time and priority, bandwidth resources being used, and the mobile terminal's trajectory and velocity. Because of these factors, the shape of a shadow cluster is usually not circular and can change over time. The center of a shadow cluster is not the geometric center of the area described by the shadow, but the cell where the mobile terminal is currently located. This cell is considered as the mobile terminal's current home cell. A bordering neighbor is a cell that shares a common border with the shadow cluster's center cell. In contrast, a non-bordering neighbor cell, although being a part of the shadow cluster, does not share a border with the shadow cluster's center cell.

Conceptually, the number and "darkness" of the shadows covering a cell reflect the amount of resources that the cell's BS needs to reserve in order to support the active mobile terminals currently in its own and in neighboring cells. With the information provided by shadow clusters, BSs can determine, for each new call request, whether the request can be supported by the wireless network. In practice, a shadow cluster is a virtual message system where BSs share probabilistic information with their neighbors on the likelihood that their active mobile terminals will move to neighbor cells (while remaining active) in the near future. With the information provided by shadow clusters, BSs project future demands and reserve resources accordingly. BSs reserve resources by denying network access to new call requests, and by "waiting" for active users to end their calls.

The decision process for the acceptance of a new call request also involves a shadow cluster. Every new call request results in the implementation of a tentative shadow cluster. BSs exchange information on their new call requests, and decide, based on this and other information, which requests should be accepted and which requests should be denied.

After a handoff, BSs within the old shadow cluster are notified about this movement, and the mobile terminal's new current BS has to assume the responsibility of supplying the appropriate information to the BSs within the new shadow cluster. BSs which were in an old shadow cluster that has just moved away must delete any entries corresponding to the active mobile terminal that established the shadow cluster, and free reserved resources if appropriate. BSs which become part of the influence region of a shadow cluster must be given appropriate information on the shadow cluster's active mobile terminal, such as the re- 


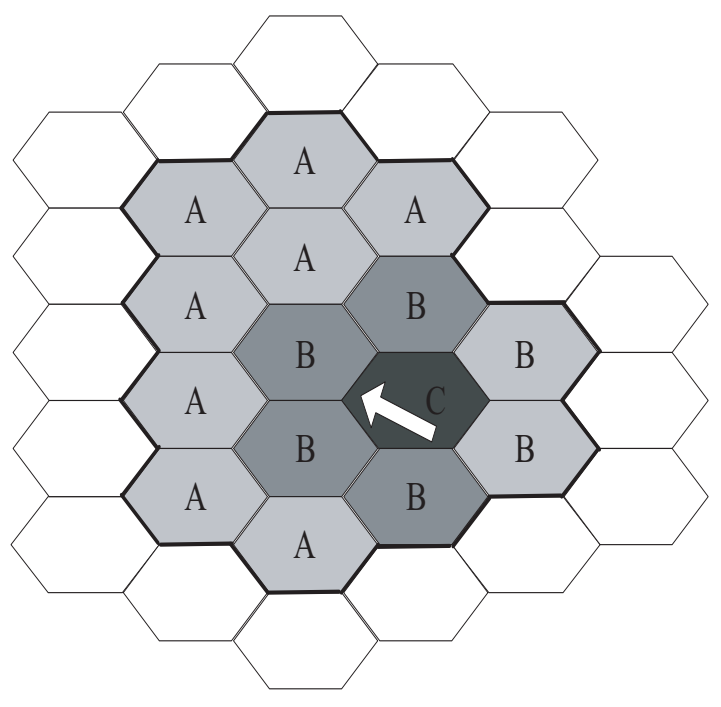

Figure 1. SCC.

spective QoS requirements, e.g., bandwidth demands, call dropping probabilities, and any other useful information such as the wireless connection's elapsed time, for the establishment of the new shadow cluster.

In our previous work, in order to deal with $\mathrm{CAC}$ in wireless cellular networks, we proposed a CAC scheme based on FL $[14,15]$. Conventional CAC schemes for wireless networks must consider some measured parameters to make the decision. However, in wireless networks due to the user mobility and varying of channel condition the measurement obtained are not accurate. Also, it is very difficult to obtain the complete statistics of the input traffic. Therefore, the CAC decision must be made based on the uncertain or inaccurate information. This is why we use FL. We implemented and evaluated the proposed system by comparing its performance with Shadow Cluster Concept (SCC) [16]. We showed that the proposed scheme could achieve a better prediction of the user behavior and a good admission decision compared with SCC.

\section{Proposed Fuzzy Admission Control System}

In our previous work, we found that the distance did not have a big effect on the CAC decision. For this reason, in the new implemented system, instead the distance we will use the priority as a new parameter for CAC decision.

The Fuzzy Logic Controller (FLC) is the main part of the proposed Fuzzy Admission Control System with Priority (FACS-P) of on-going connections and its basic elements are shown in Fig. 2. They are the fuzzifier, inference engine, Fuzzy Rule Base (FRB) and defuzzifier. As membership functions, we use triangular and trapezoidal membership functions because they are suitable for real-time oper-

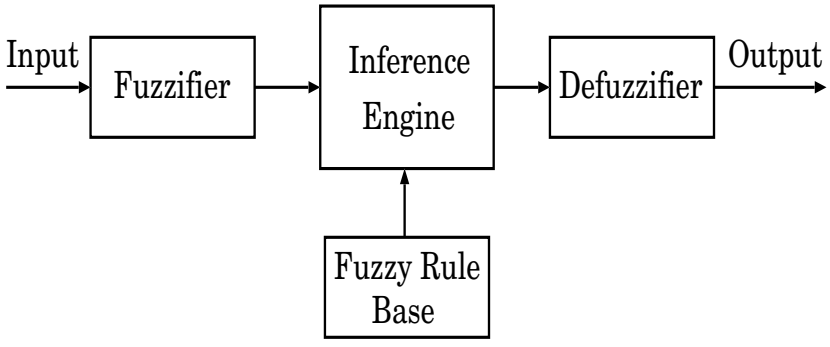

Figure 2. FLC structure.
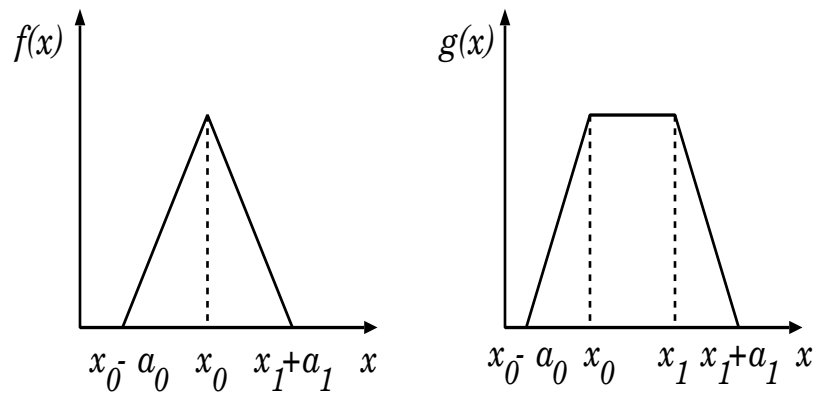

Figure 3. Triangular and trapezoidal membership functions.

ation [17-20]. The membership functions are shown in Fig. 3.

The proposed FACS-P considers the following parameters for acceptance decision: user Speed $(S p)$, user Angle $(A n)$, Service request $(S r)$, Correction value $(C v)$, Required bandwidth $(R q)$, Counter state $(C s)$, Accept or Reject decision $(A / R)$, Differentiated service $(D s)$, Real Time Counter $(R T C)$, and Non Real Time Counter (NRTC). The structure of the proposed FACS-P is shown in Fig. 4.

\subsection{FLC1 Design}

The input parameters for FLC1 are: user Speed ( $S p)$, user Angle $(A n)$, and the bandwidth of the application that we call Service request $(S r)$, while the output linguistic parameter is Correction value $(C v)$. The term sets of $S p, A n$, and $\mathrm{Sr}$ are defined respectively as:

$$
\begin{aligned}
T(S p)= & \{\text { Slow, Middle, Fast }\}=\{\text { Sl, Mi, Fa }\} \\
T(\text { An })= & \{\text { Back } 1, \text { Left } 1, \text { Left } 2, \text { Straight, Right } 1, \text { Right } 2, \\
& \text { Back } 2\}=\{\text { B } 1, \text { L } 1, \text { L } 2, \text { St }, R 1, R 2, B 2\} \\
T(\text { Sr })= & \{\text { Small, Medium, Big }\}=\{\text { Sm, Me, Bi }\} .
\end{aligned}
$$

The membership functions for input parameters of FACS-P are defined as follows: 


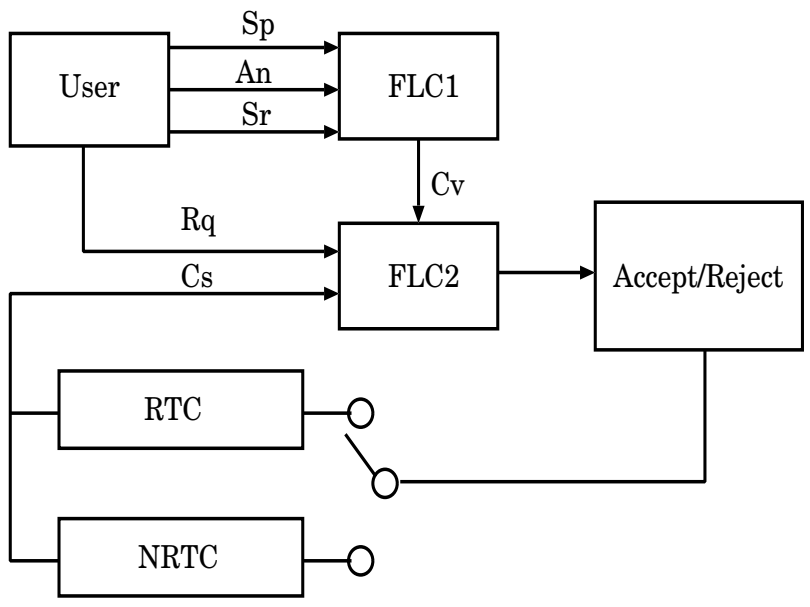

Figure 4. FACS-P model.

$$
\begin{aligned}
\mu_{S l}(S p) & \left.=f\left(S p ; S l_{0}, S l_{w 0}, S l_{w 1}\right)\right) ; \\
\mu_{M i}(S p) & =f\left(S p ; M i_{0}, M i_{w 0}, M i_{w 1}\right) ; \\
\mu_{F a}(S p) & =g\left(S p ; F a_{0}, F a_{1}, F a_{w 0}, F a_{w 1}\right) ; \\
\mu_{B 1}(A n) & =g\left(A n ; B 1_{0}, B 1_{1}, B 1_{w 0}, B 1_{w 1}\right) ; \\
\mu_{L 1}(A n) & =f\left(A n ; L 1_{0}, L 1_{w 0}, L 1_{w 1}\right) ; \\
\mu_{L 2}(A n) & =f\left(A n ; L 2_{0}, L 2_{w 0}, L 2_{w 1}\right) ; \\
\mu_{S t}(A n) & =f\left(A n ; S t_{0}, S t_{w 0}, S t_{w 1}\right) ; \\
\mu_{R 1}(A n) & =f\left(A n ; R 1_{0}, R 1_{w 0}, R 1_{w 1}\right) ; \\
\mu_{R 2}(A n) & =f\left(A n ; R 2_{0}, R 2_{w 0}, R 2_{w 1}\right) ; \\
\mu_{B 2}(A n) & =g\left(A n ; B 2_{0}, B 2_{1}, B 2_{w 0}, B 2_{w 1}\right) ; \\
\mu_{S m}(S r) & =f\left(S r ; S m_{0}, S m_{w 0}, S m_{w 1}\right) ; \\
\mu_{M e}(S r) & =f\left(S r ; M e_{0}, M e_{w 0}, M e_{w 1}\right) ; \\
\mu_{B i}(S r) & =f\left(S r ; B i_{0}, B i_{w 0}, B i_{w 1}\right) .
\end{aligned}
$$

The small letters $w 0$ and $w 1$ mean left width and right width, respectively.

The term set of the output linguistic parameter $T(C v)$ is defined as $\{$ Correction value 1, Correction value $2, \ldots$, Correction value 9$\}=\{C v 1, C v 2, \ldots, C v 9\}$. The membership functions for the output parameter $C v$ are defined as follows:

$$
\begin{aligned}
& \mu_{C v 1}(C v)=g\left(C v ; C v 1_{0}, C v 1_{1}, C v 1_{w 0}, C v 1_{w 1}\right) \\
& \mu_{C v 2}(C v)=f\left(C v ; C v 2_{0}, C v 2_{w 0}, C v 2_{w 1}\right) \\
& \mu_{C v 3}(C v)=f\left(C v ; C v 3_{0}, C v 3_{w 0}, C v 3_{w 1}\right) \\
& \mu_{C v 4}(C v)=f\left(C v ; C v 4_{0}, C v 4_{w 0}, C v 4_{w 1}\right) \\
& \mu_{C v 5}(C v)=f\left(C v ; C v 5_{0}, C v 5_{w 0}, C v 5_{w 1}\right) ; \\
& \mu_{C v 6}(C v)=f\left(C v ; C v 6_{0}, C v 6_{w 0}, C v 6_{w 1}\right) ; \\
& \mu_{C v 7}(C v)=f\left(C v ; C v 7_{0}, C v 7_{w 0}, C v 7_{w 1}\right) ; \\
& \mu_{C v 8}(C v)=f\left(C v ; C v 8_{0}, C v 8_{w 0}, C v 8_{w 1}\right) \\
& \mu_{C v 9}(C v)=g\left(C v ; C v 9_{0}, C v 9_{1}, C v 9_{w 0}, C v 9_{w 1}\right) .
\end{aligned}
$$

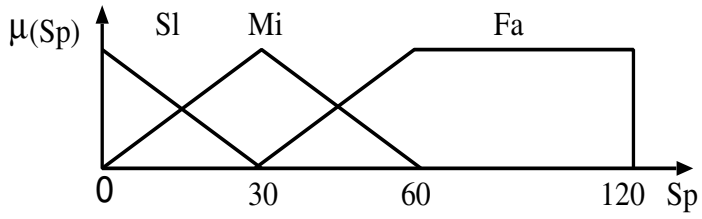

(a)

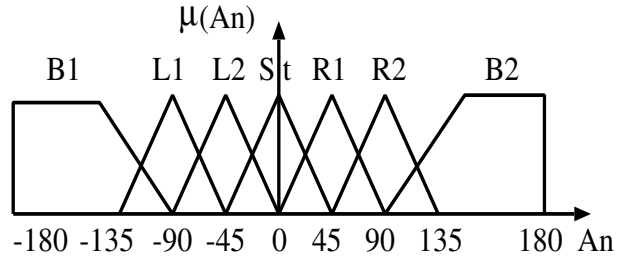

(b)

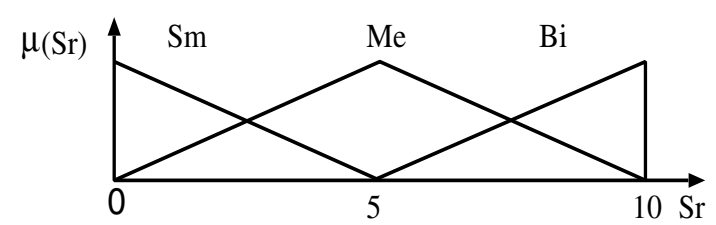

(c)

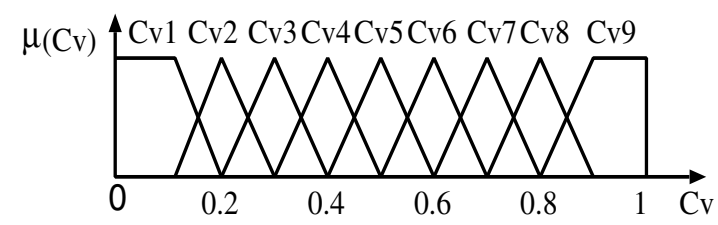

(d)

Figure 5. FLC1 membership functions.

The membership functions of FLC1 are shown in Fig. 5. The FRB forms a fuzzy set of dimensions $|T(S p)| \times$ $|T(A n)| \times|T(S r)|$, where $|T(x)|$ is the number of terms on $T(x)$. The FRB1 shown in Table 1 has 63 rules. The control rules have the following form: IF "conditions" THEN "control action".

\subsection{FLC2 Design}

For the design of FLC2, we keep the same parameters with our previous work $[14,15]$. The input parameters for FLC2 are: the output parameter of the FLC1 $(C v)$, user Request $(R q)$, and the Counter state $(C s)$, which shows the capacity of the system. While, the output linguistic parameter is the Accept/Reject decision $(A / R)$.

The term sets of $C v, R q$, and $C s$ are defined as:

$$
\begin{aligned}
& T(C v)=\{\text { Bad, Normal, Good }\}=\{\text { Bd }, \text { No, Go }\} \\
& T(R q)=\{\text { Text, Voice, } \text { Video }\}=\{\text { Tx }, \text { Vo, Vi }\} \\
& T(C s)=\{\text { Small, Middle, Full }\}=\{\text { Sa, Md }, F u\} .
\end{aligned}
$$


Table 1. FRB1.

\begin{tabular}{|c|c|c|c|c|}
\hline 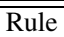 & $\overline{\mathrm{Sp}}$ & $\overline{\mathrm{An}}$ & $\mathrm{Sr}$ & $\overline{\mathrm{CV}}$ \\
\hline 0 & Sl & $\mathrm{B} 1$ & $\mathrm{Sm}$ & Cv1 \\
\hline 1 & S1 & B1 & $\mathrm{Me}$ & Cv3 \\
\hline 2 & S1 & B1 & $\mathrm{Bi}$ & $\mathrm{Cv} 2$ \\
\hline 3 & Sl & L1 & $\mathrm{Sm}$ & Cv1 \\
\hline 4 & S1 & L1 & $\mathrm{Me}$ & $\mathrm{Cv} 4$ \\
\hline 5 & S1 & L1 & $\mathrm{Bi}$ & Cv3 \\
\hline 6 & S1 & L2 & $\mathrm{Sm}$ & $\mathrm{Cv} 2$ \\
\hline 7 & $\mathrm{~S} 1$ & L2 & $\mathrm{Me}$ & Cv6 \\
\hline 8 & S1 & L2 & $\mathrm{Bi}$ & $\mathrm{Cv} 4$ \\
\hline 9 & S1 & St & $\mathrm{Sm}$ & Cv5 \\
\hline 10 & $\mathrm{~S} 1$ & St & $\mathrm{Me}$ & Cv9 \\
\hline 11 & $\mathrm{~S} 1$ & St & $\mathrm{Bi}$ & Cv7 \\
\hline 12 & $\mathrm{~S} 1$ & $\mathrm{R} 1$ & $\mathrm{Sm}$ & $\mathrm{Cv} 2$ \\
\hline 13 & $\mathrm{~S} 1$ & $\mathrm{R} 1$ & $\mathrm{Me}$ & Cv6 \\
\hline 14 & S1 & $\mathrm{R} 1$ & $\mathrm{Bi}$ & Cv4 \\
\hline 15 & $\mathrm{~S} 1$ & R2 & $\mathrm{Sm}$ & Cv1 \\
\hline 16 & $\mathrm{~S} 1$ & $\mathrm{R} 2$ & $\mathrm{Me}$ & Cv4 \\
\hline 17 & S1 & R2 & $\mathrm{Bi}$ & Cv3 \\
\hline 18 & $\mathrm{~S} 1$ & B2 & $\mathrm{Sm}$ & Cv1 \\
\hline 19 & $\mathrm{~S} 1$ & B2 & $\mathrm{Me}$ & Cv3 \\
\hline 20 & S1 & B2 & $\mathrm{Bi}$ & $\mathrm{Cv} 2$ \\
\hline 21 & $\mathrm{Mi}$ & B1 & $\mathrm{Sm}$ & Cv1 \\
\hline 22 & $\mathrm{Mi}$ & B1 & $\mathrm{Me}$ & $\mathrm{Cv} 2$ \\
\hline 23 & $\mathrm{Mi}$ & B1 & $\mathrm{Bi}$ & Cv1 \\
\hline 24 & $\mathrm{Mi}$ & L1 & $\mathrm{Sm}$ & Cv1 \\
\hline 25 & $\mathrm{Mi}$ & L1 & $\mathrm{Me}$ & Cv4 \\
\hline 26 & $\mathrm{Mi}$ & L1 & $\mathrm{Bi}$ & Cv3 \\
\hline 27 & $\mathrm{Mi}$ & L2 & $\mathrm{Sm}$ & Cv1 \\
\hline 28 & $\mathrm{Mi}$ & L2 & $\mathrm{Me}$ & Cv 5 \\
\hline 29 & $\mathrm{Mi}$ & L2 & $\mathrm{Bi}$ & Cv3 \\
\hline 30 & $\mathrm{Mi}$ & St & $\mathrm{Sm}$ & Cv8 \\
\hline 31 & $\mathrm{Mi}$ & St & $\mathrm{Me}$ & Cv9 \\
\hline 32 & $\mathrm{Mi}$ & St & $\mathrm{Bi}$ & Cv9 \\
\hline 33 & Mi & $\mathrm{R} 1$ & $\mathrm{Sm}$ & Cv1 \\
\hline 34 & $\mathrm{Mi}$ & $\mathrm{R} 1$ & $\mathrm{Me}$ & Cv5 \\
\hline 35 & $\mathrm{Mi}$ & $\mathrm{R} 1$ & $\mathrm{Bi}$ & Cv3 \\
\hline 36 & $\mathrm{Mi}$ & $\mathrm{R} 2$ & $\mathrm{Sm}$ & Cv1 \\
\hline 37 & $\mathrm{Mi}$ & $\mathrm{R} 2$ & $\mathrm{Me}$ & Cv4 \\
\hline 38 & $\mathrm{Mi}$ & $\mathrm{R} 2$ & $\mathrm{Bi}$ & Cv3 \\
\hline 39 & $\mathrm{Mi}$ & B2 & $\mathrm{Sm}$ & Cv1 \\
\hline 40 & $\mathrm{Mi}$ & B2 & $\mathrm{Me}$ & $\mathrm{Cv} 2$ \\
\hline 41 & $\mathrm{Mi}$ & B2 & $\mathrm{Bi}$ & Cv1 \\
\hline 42 & $\mathrm{Fa}$ & B1 & $\mathrm{Sm}$ & Cv1 \\
\hline 43 & $\mathrm{Fa}$ & B1 & $\mathrm{Me}$ & $\mathrm{Cv} 2$ \\
\hline 44 & $\mathrm{Fa}$ & B1 & $\mathrm{Bi}$ & Cv1 \\
\hline 45 & $\mathrm{Fa}$ & L1 & $\mathrm{Sm}$ & Cv1 \\
\hline 46 & $\mathrm{Fa}$ & L1 & $\mathrm{Me}$ & Cv3 \\
\hline 47 & $\mathrm{Fa}$ & L1 & $\mathrm{Bi}$ & $\mathrm{Cv} 2$ \\
\hline 48 & $\mathrm{Fa}$ & L2 & $\mathrm{Sm}$ & $\mathrm{Cv} 2$ \\
\hline 49 & $\mathrm{Fa}$ & L2 & $\mathrm{Me}$ & Cv5 \\
\hline 50 & $\mathrm{Fa}$ & L2 & $\mathrm{Bi}$ & Cv3 \\
\hline 51 & $\mathrm{Fa}$ & St & $\mathrm{Sm}$ & Cv9 \\
\hline 52 & $\mathrm{Fa}$ & St & $\mathrm{Me}$ & Cv9 \\
\hline 53 & $\mathrm{Fa}$ & St & $\mathrm{Bi}$ & Cv9 \\
\hline 54 & $\mathrm{Fa}$ & $\mathrm{R} 1$ & $\mathrm{Sm}$ & $\mathrm{Cv} 2$ \\
\hline 55 & $\mathrm{Fa}$ & $\mathrm{R} 1$ & $\mathrm{Me}$ & Cv5 \\
\hline 56 & $\mathrm{Fa}$ & R1 & $\mathrm{Bi}$ & Cv3 \\
\hline 57 & $\mathrm{Fa}$ & $\mathrm{R} 2$ & $\mathrm{Sm}$ & Cv1 \\
\hline 58 & $\mathrm{Fa}$ & $\mathrm{R} 2$ & $\mathrm{Me}$ & Cv3 \\
\hline 59 & $\mathrm{Fa}$ & R2 & $\mathrm{Bi}$ & $\mathrm{Cv} 2$ \\
\hline 60 & $\mathrm{Fa}$ & B2 & $\mathrm{Sm}$ & Cv1 \\
\hline 61 & $\mathrm{Fa}$ & B2 & $\mathrm{Me}$ & $\mathrm{Cv} 2$ \\
\hline 62 & $\mathrm{Fa}$ & B2 & $\mathrm{Bi}$ & Cv1 \\
\hline
\end{tabular}

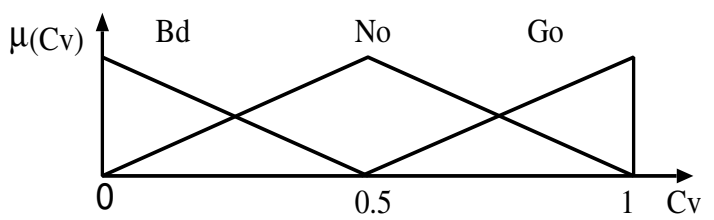

(a)

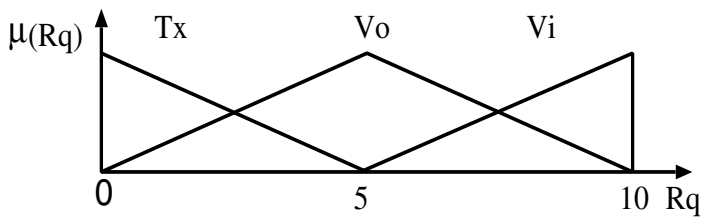

(b)

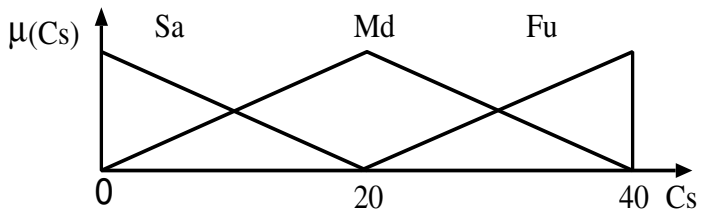

(c)

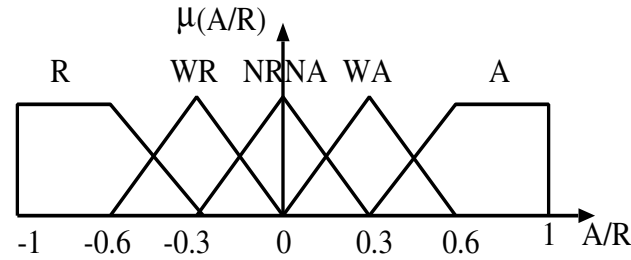

(d)

Figure 6. FLC2 membership functions.

In order to have a soft admission decision, for the output linguistic parameter $(A / R)$, we considered not only "accept" and "reject" but also "weak accept", "weak reject", and "not accept not reject" for the accept/reject decision. The membership functions for input and output linguistic parameters of FLC2 are shown in Fig. 6. The FRB2 shown in Table 2 has 27 rules.

The membership functions for input parameters of FLC2 are defined as follows:

$$
\begin{aligned}
\mu_{B d}(C v) & =f\left(C v ; B d_{0}, B d_{w 0}, B d_{w 1}\right) \\
\mu_{N o}(C v) & =f\left(C v ; N o_{0}, N o_{w 0}, N o_{w 1}\right) ; \\
\mu_{G o}(C v) & =f\left(C v, G o_{0}, G o_{w 0}, G o_{w 1}\right) ; \\
\mu_{T x}(R q) & =f\left(R q ; T x_{0}, T x_{w 0}, T x_{w 1}\right) ; \\
\mu_{V o}(R q) & =f\left(R q ; V o_{0}, V o_{w 0}, V o_{w 1}\right) ; \\
\mu_{V i}(R q) & =f\left(R q ; V i_{0}, V i_{w 0}, V i_{w 1}\right) ; \\
\mu_{S a}(C s) & =f\left(C s ; S a_{0}, S a_{w 0}, S a_{w 1}\right) ; \\
\mu_{M d}(C s) & =f\left(C s ; M d_{0}, M d_{w 0}, M d_{w 1}\right) \\
\mu_{F u}(C s) & =f\left(C s ; F u_{0}, F u_{w 0}, F u_{w 1}\right)
\end{aligned}
$$


Table 2. FRB2.

\begin{tabular}{|l|l|l|l|l|}
\hline \hline Rule & Cv & R & Cs & A/R \\
\hline 0 & Bd & Tx & Sa & A \\
1 & Bd & Tx & Md & NRNA \\
2 & Bd & Tx & Fu & NRNA \\
3 & Bd & Vo & Sa & A \\
4 & Bd & Vo & Md & NRNA \\
5 & Bd & Vo & Fu & WR \\
6 & Bd & Vi & Sa & WA \\
7 & Bd & Vi & Md & NRNA \\
8 & Bd & Vi & Fu & WR \\
9 & No & Tx & Sa & A \\
10 & No & Tx & Md & NRNA \\
11 & No & Tx & Fu & NRNA \\
12 & No & Vo & Sa & A \\
13 & No & Vo & Md & NRNA \\
14 & No & Vo & Fu & NRNA \\
15 & No & Vi & Sa & WA \\
16 & No & Vi & Md & NRNA \\
17 & No & Vi & Fu & NRNA \\
18 & Go & Tx & Sa & A \\
19 & Go & Tx & Md & A \\
20 & Go & Tx & Fu & NRNA \\
21 & Go & Vo & Sa & A \\
22 & Go & Vo & Md & A \\
23 & Go & Vo & Fu & WR \\
24 & Go & Vi & Sa & A \\
25 & Go & Vi & Md & A \\
26 & Go & Vi & Fu & R \\
\hline
\end{tabular}

The term set of the output linguistic parameter $T(A / R)$ is defined as $\{$ Reject, Weak Reject, Not Reject Not Accept, Weak Accept, Accept $\}$. We write for short as $\{\mathrm{R}, \mathrm{WR}$, NRNA, WA, A $\}$. The membership functions for the output parameter $A / R$ are defined as follows:

$$
\begin{aligned}
\mu_{R}(A / R) & =g\left(A / R ; R_{0}, R_{1}, R_{w 0}, R_{w 1}\right) ; \\
\mu_{W R}(A / R) & =f\left(A / R ; W R_{0}, W R_{w 0}, W R_{w 1}\right) ; \\
\mu_{N R N A}(A / R) & =f\left(A / R ; N R N A_{0}, N R N A_{w 0}, N R N A_{w 1}\right) ; \\
\mu_{W A}(A / R) & =f\left(A / R ; W A_{0}, W A_{w 0}, W A_{w 1}\right) ; \\
\mu_{A}(A / R) & =g\left(A / R ; A_{0}, A_{1}, A_{w 0}, A_{w 1}\right) .
\end{aligned}
$$

\section{Simulation Results}

The simulation were carried out in Linux Fedora Core5 computer. We considered the following parameters for simulations: the user speed was from 0 to $120 \mathrm{~km} / \mathrm{h}$, the user

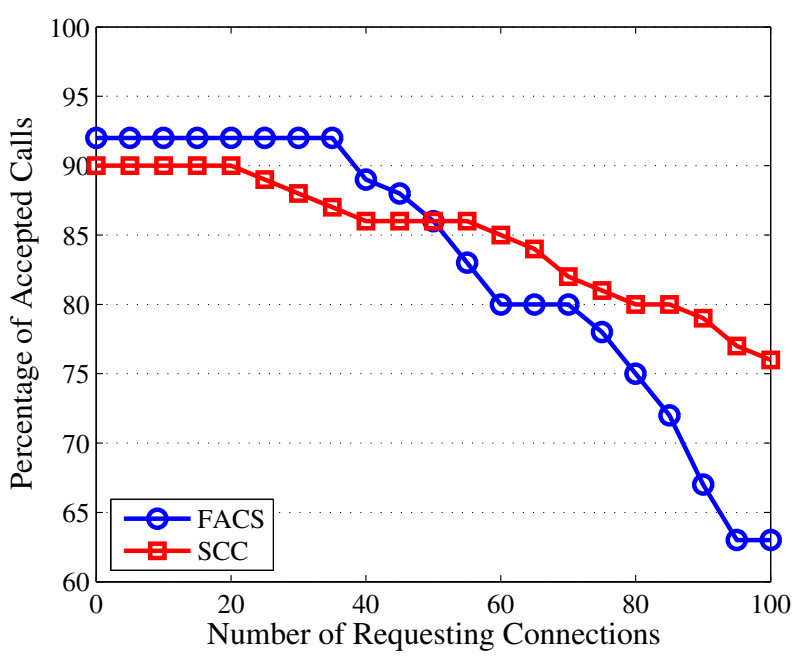

Figure 7. Performance of FACS and SCC.

direction was changed from -180 degree to +180 degree, the service request for text, voice and video was considered $70 \%, 20 \%$, and $10 \%$, respectively. The requested size was 1, 5 and 10 Bandwidth Units (BU) for text, voice and video, respectively. The bandwidth of the BS was considered 40 BU.

In Fig. 7, we show the performance of our previous FACS and SCC. When the number of requesting connections is less than 50, the percentage of accepted calls for proposed system is higher than SCC. However, when the number of requesting connections is larger than 50 , the proposed system accepts less number of connections. This is because, the proposed system guarantees the QoS of ongoing calls and requesting connections. When the number of requesting connections is less than 50 (the case when there is enough $\mathrm{BU}$ ), the proposed system make a better allocation of the resources compared with SCC.

In Fig. 8 is shown the relation between percentage of accepted calls versus number of requesting connections. In this simulation, we consider the user speed as a parameter. From the simulation results can be seen that with the increase of the user speed, the percentage of the number of the accepted calls is increased. This happens because with the increase of the user speed, the user direction can not be changed easily, this results in a better prediction of the user direction and the network resources are used better. On the other hand, when the user speed is slow, the prediction of the user direction becomes difficult, which results in a small percentage of the accepted calls.

In Fig. 9, we consider the angle as parameter. We show the simulation results for different angels from 0 to 90 degree. When the user angle is small, the percentage of accepted calls is higher and is decreased with the increase of 


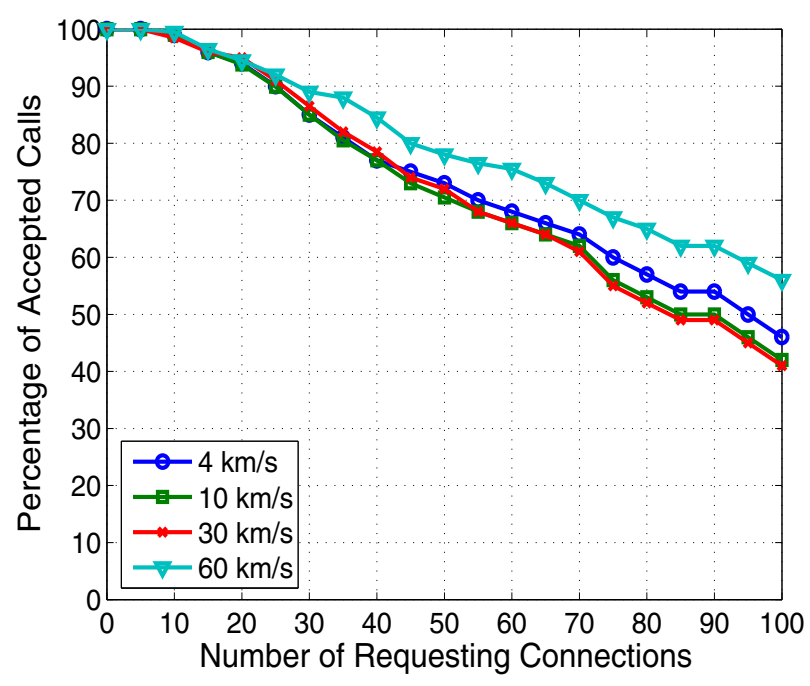

Figure 8. Percentage of number of accepted calls vs. number of requesting connections for different speed values (FACS-P).

the number of requesting connections. With the increase of the angle, the user is going far from the BS, so there is not need to allocate the bandwidth for this user. This is why the percentage of the number of accepted calls is decreased with the increase of the angle value. When the angle is more than 90 degree, the percentage of accepted calls is almost zero. For this reason, we did not show in this figure.

In order to evaluate the performance of the proposed system, we compare its performance with our previous FACS. The simulation results are shown in Fig. 10. When the number of requesting connections is less than 25 , the percentage of accepted calls for proposed system is higher than our previous system. However, when the number of requesting connections is larger than 25 , the proposed system accepts less number of connections. This is because, the proposed system guarantees the QoS of on-going calls, thus decreasing the acceptance ratio of the requesting connections.

\section{Conclusions}

In this paper, we proposed a fuzzy based admission control system with priority for on-going connections (called FACS-P). We evaluated the performance of the proposed system for different scenarios. We also compared the performance of the proposed system FACS-P with our previous system FACS.

From the simulations results, we conclude as follows:

- with the increase of the user speed, the percentage of the number of the accepted calls is increased;

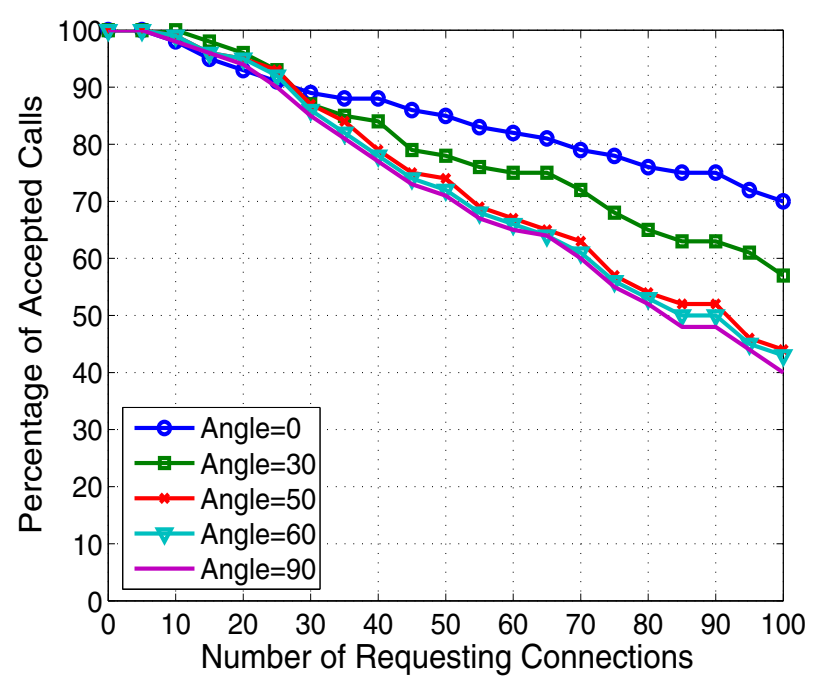

Figure 9. Percentage of number of accepted calls vs. number of requesting connections for different angle values (FACS-P).

- when the user speed is slow, the prediction of the user direction becomes difficult, which results in a small percentage of the accepted calls;

- when the user angle is small, the percentage of accepted calls is higher and is decreased with the increase of the number of requesting connections;

- the proposed system keeps a higher QoS of on-going connections.

In this work, we considered only the priority of on-going connections. In the future, we would like to consider also the priority of requesting connections.

\section{References}

[1] Y. Fang, and Y. Zhang, "Call Admission Control Schemes and Performance Analysis in Wireless Mobile Networks", IEEE Trans. on Vehicular Technology, Vol. 51, No. 2, pp. 371-382, March 2002.

[2] W. Wang, X. Wang, and A. A. Nilsson, "EnergyEfficient Bandwidth Allocation in Wireless Networks: Algorithms, Analysis, and Simulations", IEEE Transactions on Wireless Communications, Vol 5, No. 5, pp. 1103-1114, May 2006.

[3] D. Z. Deniz and N. O. Mohamed, "Performance of CAC Strategies for Multimedia Traffic in Wireless Networks", IEEE Journal on Selected Areas in Commun., Vol. 21, No. 10, pp. 1557-1565, December 2003. 


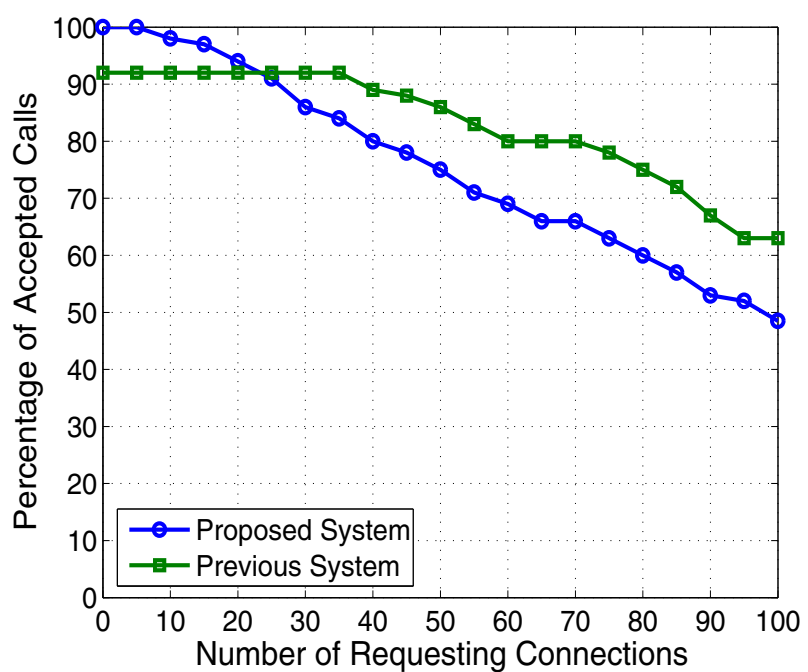

Figure 10. Performance of proposed FACS-P with FACS.

[4] L. Barolli, A. Koyama, T. Yamada, S. Yokoyama, T. Suganuma, N.Shiratori, "A Fuzzy Admission Control Scheme and Its Performance Evaluation", IPSJ Journal, Vol. 42, No. 12, pp. 3213-3221, December 2001.

[5] L. Barolli, A. Koyama, T. Suganuma and N. Shiratori, "A Genetic Algorithm Based QoS Routing Method for Multimedia Communications Over High-Speed Networks", IPSJ Journal, Vol. 44, No. 2, pp. 544-552, February 2003.

[6] B. Bensaou, S. T. C. Lam, H-W. Chu, D. H. K. Tsang, "Estimation of the Cell Loss Ratio in ATM Networks with a Fuzzy System and Application to Measurement-Based Call Admission Control", IEEE/ACM Trans. on Networking, Vol. 5, No. 4, pp. 572-584, August 1997.

[7] I. Habib, (Ed.), "Neurocomputing in High-Speed Networks", IEEE Commun. Magazine, Special Issue, Vol. 33, No. 10, 1995.

[8] L. Barolli, A. Koyama, T. Suganuma, N. Shiratori, "GAMAN: A GA Based QoS Routing Method for Mobile Ad-hoc Networks", Journal of Interconnection Networks (JOIN), Vol. 4, No. 3, pp. 251-270, September 2003.

[9] A. Koyama, L. Barolli, G. Capi, B. O. Apduhan, J. Arai, A.Durresi, ”An Efficient Multi-Purpose Optimization Method for QoS Routing Using Genetic Algorithm", Journal of Interconnection Networks (JOIN), Vol. 5, No. 4, pp. 409-428, December 2004.
[10] M. Ikeda, L. Barolli, A. Koyama, A. Durresi, G. De Marco, J. Iwashige, "Performance Evaluation of an Intelligent CAC and Routing Framework for Multimedia Applications in Broadband Networks", Journal of Computer and System Science (JCSS), Vol. 72, Issue 7, pp. 1183-1200, November 2006.

[11] L. Barolli, M. Ikeda, G. De Marco, A. Durresi, A. Koyama, J. Iwashige, "A Search Space Reduction Algorithm for Improving the Performance of a GA-based Routing Method in Ad-Hoc Network", International Journal of Distributed Sensor Networks (IJDSN), Vol. 3, No. 1, pp. 41-57, January 2007.

[12] L. Barolli, gA Speed-Aware Handover System for Wireless Cellular Networks Based on Fuzzy Logich, Mobile Information Systems (MIS), Vol. 4, No. 1, pp. 1-12, 2008.

[13] L. Barolli, J. Anno, F. Xhafa, A. Durresi, A. Koyama, "A Context-Aware Fuzzy-Based Handover System for Wireless Cellular Networks and Its Performance Evaluation", Journal of Mobile Multimedia (JMM), Vol. 4. No. 3/4, pp. 241-258, October 2008.

[14] L. Barolli, F. Xhafa, A. Durresi, A. Koyama, "A Fuzzy-based Call Admission Control System for Wireless Cellular Networks", Proc. of MNSA2007/ICDCS-2007, Toronto, Canada, CD-ROM, 8 pages, June 2007.

[15] L. Barolli, "An Intelligent Call Admission Control System for Wireless Cellular Networks Based on Fuzzy Logic", Journal of Mobile Multimedia (JMM), Vol. 3. No. 4, pp. 331-346, December 2007.

[16] D. A. Levine, I. F. Akyildiz, M. Naghshineh, "A Resource Estimation and Call Admission Algorithm for Shadow Cluster Concept", IEEE/ACM Transactions on Networking, Vol. 5, No. 1, pp. 1-12, 1997.

[17] D. Dubois, H. Prade, R. Yager, (Eds.), "Fuzzy Sets for Intelligent Systems", Morgan Kaufman Publishers, 1993.

[18] L. A. Zadeh, "Fuzzy Logic, Neural Networks, and Soft Computingh, Communication of ACM, Vol. 37, No. 3, pp. 77-84, March 1994.

[19] R. R. Yager, and L. A. Zadeh, editors, "An Introduction To Fuzzy Logic Applications In Intelligent Systems", Kluwer Academic Publishers, 1992.

[20] H. J. Zimmermann, "Fuzzy Set Theory-and Its Applications", Kluwer Academic Publishers, Second Revised Edition, 1991. 